\title{
Effect of the Reverse Shock on the Parameters of the Observed X-ray Emission during the 1998 Outburst of CI Camelopardalis
}

\author{
E. V. Filippova ${ }^{1,2 *}$, M. G. Revnivtsev ${ }^{1,3}$, and A. A. Lutovinov ${ }^{1}$ \\ ${ }^{1}$ Space Research Institute, Russian Academy of Sciences, Profsoyuznaya ul. 84/32, Moscow, 117997 Russia \\ ${ }^{2}$ Max-Planck-Institut für Astrophysik, Karl-Schwarzschild-Straße 1, Postfach 1317, D-85741 Garching, \\ Germany \\ ${ }^{3}$ Excellence Cluster Universe, Technische Universität München, James-Franck-Straße 1, D-85748 Garching, \\ Germany \\ Received February 10, 2009
}

\begin{abstract}
Based on the model of interaction between spherically symmetric expanding matter and the external medium, we have estimated the parameters of the matter heated by the shock that was produced in the envelope ejected by the explosion of a classical nova during its interaction with the stellar wind from the optical companion. Using this model, we have shown that the matter ejected during the outburst in the system CI Camelopardalis had no steep velocity gradients and that the reverse shock could heat the ejected matter only to a temperature of $\sim 0.1 \mathrm{keV}$. Therefore, this matter did not contribute to the mean temperature and luminosity of the system observed in the energy range $3-20 \mathrm{keV}$.
\end{abstract}

PACS numbers: 97.30.Qt; 97.80.Gm; 95.30.Lz

DOI: $10.1134 / \mathrm{S} 1063773709100053$

Key words: classical novae, $X$-ray emission, numerical simulations.

\section{INTRODUCTION}

Classical nova outbursts can be accompanied by emission in both the standard $(1-10 \mathrm{keV})$ and hard $(>20 \mathrm{keV}) \mathrm{X}$-ray energy bands. Matter heated by the shock that was produced by a high-velocity $\left(\sim 1000-4000 \mathrm{~km} \mathrm{~s}^{-1}\right)$ expansion of the envelope of a white dwarf is believed to be the source of this X-ray emission. In the system CI Camelopardalis (CI Cam), the optical companion is a B[e]-type B4 III-V star (Barsukova et al. 2006) with a strong stellar wind (Robinson et al. 2002; Filippova et al. 2008), which produces a dense circumstellar medium around the white dwarf. Filippova et al. (2008) (hereafter Paper I) showed that, in this case, a shock is generated by the envelope expansion in the circumstellar medium, which could heat a large amount of stellar wind matter up to $10-20 \mathrm{keV}$ sufficient to produce a high $\mathrm{X}$-ray luminosity.

In the envelope itself, a shock (or initially a reverse rarefaction wave that will transform into a reverse shock as the envelope expands) will also be generated. Under certain conditions, this shock can also heat the matter to high temperatures. For example, it follows from the analytical calculations by Chevalier (1982)

\footnotetext{
*E-mail: kate@iki.rssi.ru
}

and Nadyozhin (1985) that when the ejected matter interacts with a constant-density medium, the temperature at the reverse shock for a power in the ejected matter density profile of $\sim 6-8$ can be lower than the temperature at the forward shock only by a factor of $\sim 2-4$.

A schematic view of the system of shocks produced by the interaction of ejected matter with the circumstellar medium is shown in Fig. 1 (the outer boundary of the envelope is a contact discontinuity). In general, the formation and the law of motion of the reverse shock depend on the matter velocity and pressure distributions inside the envelope. However, at present, there is no complete model that would consistently describe the evolution of the profiles of these parameters in the matter ejected by the explosion of a classical nova (see, e.g., a review of theoretical models in Friedjung (2002)). On the basis of numerical calculations and their comparison with observational data during a classical nova explosion, two mechanisms of matter ejection at the initial time are suggested: under the action of thermal pressure and through a shock wave. The consequences of the ejection of matter through these mechanisms were considered by Sparks (1969). He showed that in the case of pressure-driven expansion, the envelope has a very shallow matter velocity gradient, while in the 
case of shock-driven ejection of matter, it expands with a steep velocity gradient. At later expansion stages, after the maximum optical brightness of the nova, the expansion of matter is described by the model of an optically thick wind (Kato and Hachisu 1994; Hauschildt et al. 1994). There also exist theories predicting that shortly after the maximum optical brightness, the velocities of the envelope layers closer to the white dwarf are higher than those of the outer layers (McLaughlin et al. 1947, 1964).

An example of the possible development of a classical nova explosion was given by Prialnik (1986), who calculated a complete cycle of the evolution of a classical nova explosion, from the phase of accretion to its resumption; the conditions for the generation of a shock wave in the envelope were met. According to these calculations, within the first half an hour ( 2000 s) after the thermonuclear explosion, the white dwarf photosphere expands to $\sim 10 R_{\odot}$ due to the shock breakout. Within the next $\sim 4 \mathrm{~h}$, the envelope expands even more due to the radiation pressure from the white dwarf surface, with the optical flux from the system reaching its maximum. It follows from observations that the characteristic time it takes for the optical flux to reach its maximum for most classical novae is $<3$ days, but exceptions are also observed; for example, during the outburst of Nova LMC 1991 this time was $\geq 13$ days (Schwarz et al. (2001) and references therein). According to calculations, the outer layers of the envelope at this time expand with constant velocities, which increase toward the outer boundary and reach $\sim 3800 \mathrm{~km} \mathrm{~s}^{-1}$ at it. This part of the envelope ceases to be connected with the white dwarf and expands by inertia, interacting only with the circumstellar medium. The remaining part of the envelope initially contracts under the gravitational force and, after some time, again begins to expand under radiation pressure in the regime of an optically thick wind.

It was shown in Paper I that the main peculiarities of the behavior of the light curve and radiation temperature during the X-ray outburst of CI Cam in the first (spherically symmetric) approximation could be described in terms of the radiation model of stellar wind matter heated by the forward shock produced in a classical nova explosion. In this model, the envelope is ejected from the white dwarf due to explosive thermonuclear burning, which already on $0.1-0.5$ day after the explosion onset has an expansion velocity of $\sim 2700 \mathrm{~km} \mathrm{~s}^{-1}$ and flies under the action of an external force, for example, the radiation pressure from the white dwarf, with a constant velocity for the first $\sim 1-1.5$ days. Subsequently, the envelope probably becomes transparent and decelerates, interacting with the matter of the stellar wind from the optical companion. Based on a comparison of the observed

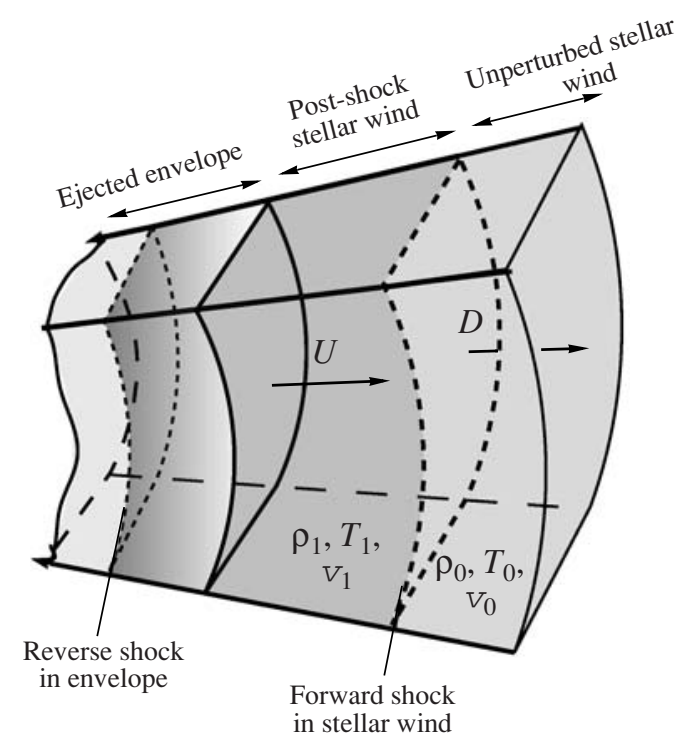

Fig. 1. Scheme of the shocks produced by the interaction of the expanding envelope with the circumstellar medium: $U$ is the velocity of the contact discontinuity or the envelope (depending on the model considered) and $D$ is the velocity of the forward shock.

rise in luminosity with the theoretical dependence, we estimated the stellar wind density near the white dwarf to be $n_{0}\left(r<r_{c}\right) \sim 8.6 \times 10^{9} d_{2 \mathrm{kpc}} U_{2700}^{-3 / 2} \mathrm{~cm}^{-3}$, which transforms into the law $n_{0} \sim r^{-2}$ at $r>r_{c}=$ $1.9 \times 10^{13} \mathrm{~cm}$. In the simplest model, this stellar wind density distribution corresponds to a mass loss rate of the optical star $\sim(1-2) \times 10^{-6} M_{\odot} \mathrm{yr}^{-1}$. The observed time dependence of the temperature of the emitting matter at late envelope expansion stages allowed us to constrain the mass of the ejected envelope based on our model, $10^{-7}-10^{-6} M_{\odot}$. Note that in this model, the processes in the envelope itself were disregarded; in our calculations, we used a finitemass piston as the envelope.

In this paper, we calculated the contribution from the emission of the ejected envelope matter heated by the reverse shock to the observed radiation temperature and luminosity of CI Cam during its X-ray outburst in 1998.

\section{NUMERICAL CALCULATIONS}

To model the reverse shock, we used a numerical scheme described in Paper I: a one-dimensional, spherically symmetric code in Lagrangian coordinates with a staggered mesh (the cell radius, velocity, and mass are determined at the cell boundaries, while the density, pressure, and internal energy are determined at the cell centers).

At the initial time, the outer boundary of the envelope was placed at a distance of $10^{12} \mathrm{~cm}$. The density 

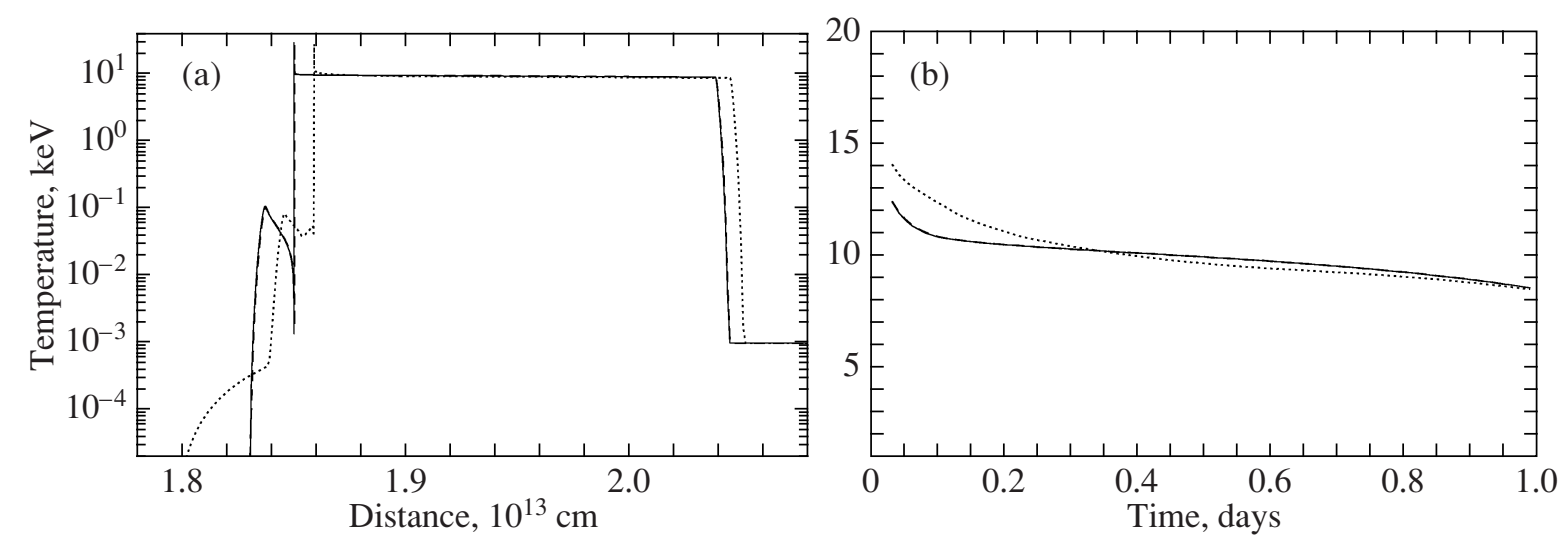

Fig. 2. (a) Temperature profiles in the envelope and stellar wind on 0.8 day after the explosion for various initial envelope matter temperatures; the solid, dashed, and dotted lines correspond to $10^{3}, 10^{4} \mathrm{~K}$ (in both cases, the contact discontinuity is at a distance of $\sim 1.85 \times 10^{13} \mathrm{~cm}$ ), and $10^{6} \mathrm{~K}$ (the contact discontinuity is at a distance of $\sim 1.86 \times 10^{13}$ cm), respectively; (b) time dependence of the mean temperature for these cases.

of the circumstellar medium was specified as follows: $n_{0}=8 \times 10^{9} \mathrm{~cm}^{-3}$ at $r<r_{c}$ and $n_{0} \sim r^{-2}$ at $r>$ $r_{c}$, where $r_{c}=1.9 \times 10^{13} \mathrm{~cm}$. The initial cell size was $\Delta r=10^{10} \mathrm{~cm}$. The velocity of the matter at the inner and outer boundaries was specified by a timeindependent constant.

As in Paper I, we took into account the radiative cooling of the matter heated by the shocks in an optically thin regime.

As was shown in Paper I, the matter behind the shocks is a multitemperature plasma in the sense that the plasma temperature is nonuniform along the radius. Consequently, the radiation temperature that we measure based on X-ray observations is an average quantity and it may not be equal to the temperature at the shock front. Therefore, to obtain the calculated mean temperature, we used the same averaging procedure as that for observations. We calculated the ratio of the fluxes in the $3-5$ and 5$20 \mathrm{keV}$ energy bands, which, in turn, corresponds to a certain temperature in the radiation model of a singletemperature, optically thin plasma. The method is described in more detail in Paper I.

\section{Effect of the Matter Temperature in the Envelope on the Calculated Parameters}

The temperature or pressure of the envelope matter is a parameter that, in general, can affect the formation and propagation of shocks after the decay of an arbitrary discontinuity.

To understand what the temperature and pressure distributions in the ejected envelope are, we can turn to actual observations of classical novae. Two outbursts of novae (Cyg 1992 and LMC 1991) that were observed before the maximum optical brightness was reached and for which the radiation temperature was measured are known to date. However, the effective radiation temperature obtained in such an analysis of observations is not a good indicator of the physical temperature in the envelope (Hauschildt et al. 1994; Schwarz et al. 2001). Nevertheless, since the emission from the envelope matter has a maximum in the ultraviolet, we may assert that the matter temperature does not exceed $\sim 0.1 \mathrm{keV}$ in order of magnitude.

To answer the question of how the envelope matter temperature affects the propagation of shock waves, we performed calculations with the following initial parameters of the matter in the envelope: the density is constant along the radius (the envelope mass was $\left.10^{-6} M_{\odot}\right)$, the velocity is also constant along the radius and equal to $2700 \mathrm{~km} \mathrm{~s}^{-1}$, and we considered several matter temperatures: $10^{3}, 10^{4}$, and $10^{6} \mathrm{~K}$.

Figure 2a shows the matter temperature profiles in the envelope and stellar wind on 0.8 day after the onset of expansion: the dotted, dashed, and solid lines correspond to $T=10^{6}, 10^{4}$, and $10^{3} \mathrm{~K}$, respectively. It follows from the figure that the expected range of envelope matter temperatures affects weakly the propagation dynamics and strength of the forward shock and leads to unimportant differences in the time dependence of the mean radiation temperature (Fig. 2b). Therefore, below, the initial temperature of the envelope matter in our calculations was set equal to a constant, $10^{4} \mathrm{~K}$, unless stated otherwise.

\section{TRANSFORMATION OF THE REVERSE RAREFACTION WAVE INTO A REVERSE SHOCK}

As was noted in Paper I, the interaction of the ejected envelope with the circumstellar medium can 


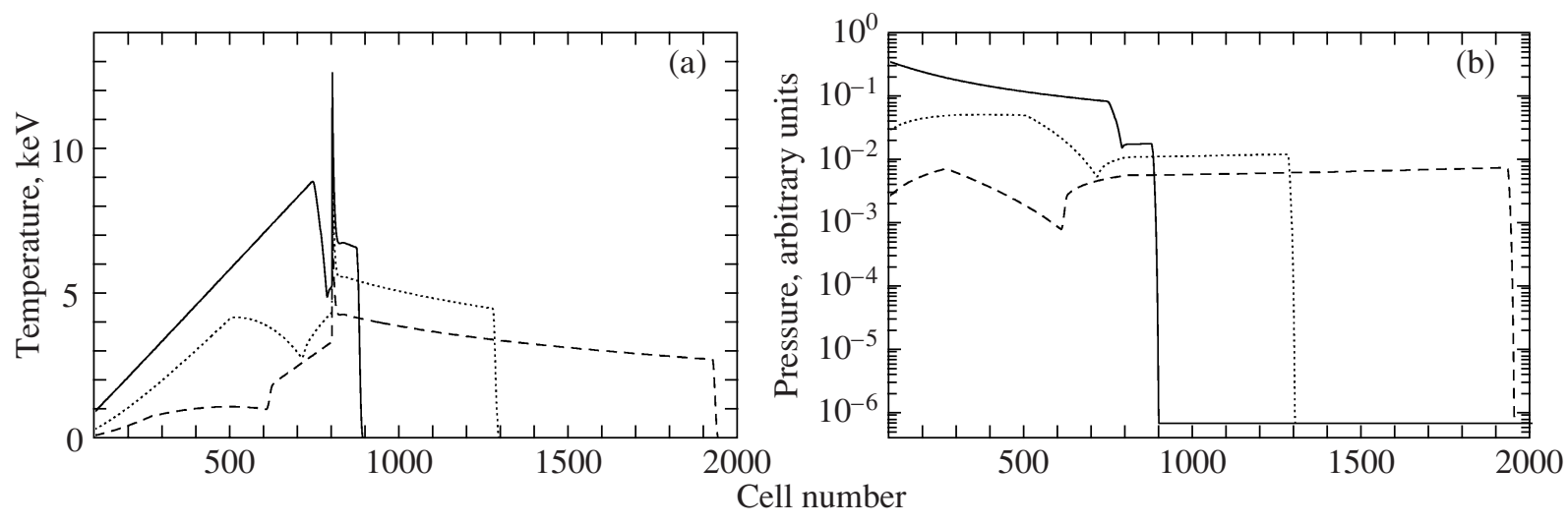

Fig. 3. (a) Temperature profiles in the ejected matter and stellar wind at various times; the cell number is along the $X$ axis, the contact boundary is located on cell no. 800. (b) Pressure profiles at the same times.

give rise to a reverse rarefaction wave at the very outset. The instant of the subsequent transformation of the reverse rarefaction wave into a reverse shock depends on the radial distribution of envelope matter parameters (such as the velocity and pressure). In the same paper, we made very simple estimates of the conditions under which the reverse rarefaction wave is generated and the time when it transforms into a reverse shock. It follows from these estimates that the reverse rarefaction wave transforms into a shock almost immediately.

For a clear demonstration of this phenomenon, we performed calculations in which this transformation could be traced in more detail. It should be noted that the parameters specified as the initial conditions bear no relation to the actual values: for example, in order that the reverse rarefaction wave could recede noticeably from the contact discontinuity, the matter temperature at the outer boundary of the envelope was set equal to $10 \mathrm{keV}$, but in order that the disturbances arising at the inner boundary have no time during the calculations to propagate over the entire envelope, the matter temperature was specified by a linear function of the radius and was $\sim 0.01 \mathrm{keV}$ at the inner boundary; the energy losses through radiation were disregarded. The expansion velocity of the envelope matter was $600 \mathrm{~km} \mathrm{~s}^{-1}$.

Figure 3 shows the temperature and pressure profiles obtained in this model in the interacting region at various times. We clearly see how the rarefaction wave is generated (solid line) and how it transforms into a reverse shock (in the profiles drawn by the dashed line, the reverse shock is seen clearly). The cell number is along the $X$ axis, with the contact boundary being located on cell no. 800; the forward shock propagates through the stellar wind rightward; the rarefaction wave propagates through the ejected matter leftward and transforms into a shock.

\section{HOMOLOGOUS ENVELOPE EXPANSION $(v \sim r)$}

Classical nova explosions resemble in mechanism type Ia supernova (SN Ia) explosions, thermonuclear explosions of white dwarfs (Woosley and Weaver 1986). However, in the former case, the explosion energy and, hence, the kinetic energy of the ejected envelope are much lower than those in the latter case. For classical nova explosions and supernovae, the kinetic energy of the ejected envelope is estimated to be $\sim 10^{44}-10^{45}$ (Starrfield et al. 1976) and $\sim 10^{51} \mathrm{erg}$ (Khokhlov et al. 1993), respectively.

Numerical calculations show that a homologous expansion of the ejected matter during an SN Ia explosion is established in $\sim 10 \mathrm{~s}$ (Röpke (2005) and references therein). Dwarkadas et al. (1998) provided the density profiles of the ejected matter for several $\mathrm{SN}$ Ia explosion models used to describe the observational data. They also showed that the density profiles could be described both by a power law with an exponent of 7 (nevertheless, the exponent can often be different from 7) and by an exponential law $\rho \sim$ $\exp ^{-v / v_{0}}$.

Chevalier (1982) and Nadyozhin (1985) provided self-similar solutions for the decay of an arbitrary discontinuity in the case of a spherically symmetric homologous envelope expansion with exponents in the density distribution $p>5$ into a constant-density external medium. It follows from these solutions that when the reverse shock is produced, the temperature at the forward shock decreases with time. For example, for $p=5.4$, the time dependence of the temperature at the forward shock is $T_{1} \sim\left(t / t_{\min }\right)^{-10 / 9}$ (Nadyozhin 1985). For an envelope with a mass of $10^{-6} M_{\odot}$, its kinetic energy is $10^{44} \mathrm{erg}$ and the density of the matter of the external medium is $8 \times 10^{9} \mathrm{~cm}^{-3}$, the time $t_{\min } \sim 9000 \mathrm{~s}$; we then find from the formula that in the first day of expansion, the temperature 


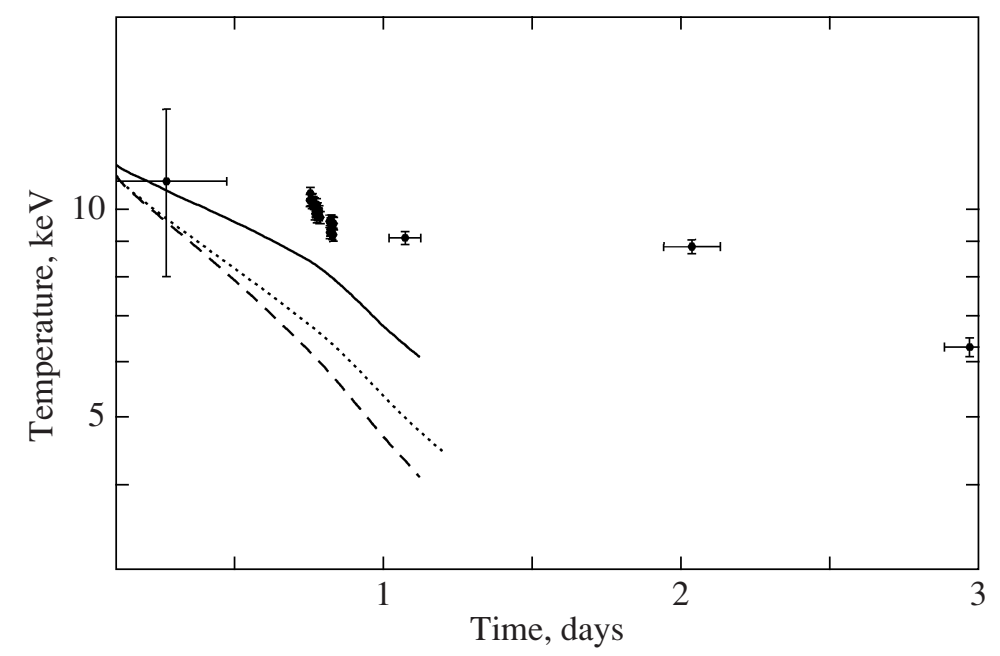

Fig. 4. Time dependence of the mean temperature of the matter behind the forward shock for a homologous expansion of the matter in the envelope: the solid line correspond to the model with a mass of the matter in the envelope having a velocity $>2000 \mathrm{~km} \mathrm{~s}^{-1}$ of $2 \times 10^{-5} M_{\odot}$ and $p=3$; the dashed line corresponds to $10^{-6} M_{\odot}$, the exponent in the density distribution of the ejected matter is $p=3$; the dotted line corresponds to $2 \times 10^{-5} M_{\odot}, p=15$.

at the forward shock will fall by a factor of $\sim 10$, which will lead to a decrease in the mean radiation temperature.

To understand how the homologous envelope expansion in our problem affects the behavior of the mean radiation temperature, we performed the following numerical calculations. First, we considered the case where the mass loss rate from the white dwarf as a result of the shock breakout was constant. The exponent in the radial density distribution of the ejected matter is then $p=3$. Since the velocity of the ejected matter decreases with decreasing radius, only the high-velocity outer layers of the envelope are actually of interest for the generation of a forward shock and energy estimations for the Sedov phase. For our subsequent estimations of the kinetic energy and mass of the envelope, we took a lower velocity limit of $2000 \mathrm{~km} \mathrm{~s}^{-1}$.

We performed our calculations for two models: the mass of the matter ejected with a velocity $>2000 \mathrm{~km} \mathrm{~s}^{-1}$ is $\sim 10^{-6} M_{\odot}$ and $\sim 2 \times 10^{-5} M_{\odot}$. The kinetic energy of the envelope is $E_{\text {kin }, v>2000} \sim$ $7 \times 10^{43}$ erg in the former case and $E_{\text {kin, } v>2000} \sim$ $1.2 \times 10^{45} \mathrm{erg}$ in the latter case. In calculating the mass, we used the cosmic abundance of the matter. However, since the heavy-element abundance in the ejected envelope is believed to be higher than the cosmic one (Starrfield et al. 1976; Yaron et al. 2005), the mass obtained and energy are lower limits.

Figure 4 shows the time dependence of the mean temperature of the matter behind the forward shock for these two cases (the solid and dashed lines correspond to masses of the matter with a velocity
$>2000 \mathrm{~km} \mathrm{~s}^{-1}$ of $2 \times 10^{-5} M_{\odot}$ and $10^{-6} M_{\odot}$, respectively). In Paper I, we estimated the explosion energy required to obtain the observed time dependence of the mean temperature of the matter at late expansion stages, when the shock enters the Sedov regime (the regime in which the shock "forgets" the details of its formation and evolves self-similarly). ${ }^{1}$ According to these calculations, the energy should be $\sim(5-$ 10) $\times 10^{43} \mathrm{erg}$. Thus, a further increase in the mass of the ejected matter in the model with a homologous expansion will lead to a higher (compared to the observations) radiation temperature on 4-10 days of expansion.

In Hauschildt et al. (1994) and Schwarz et al. (2001), who investigated the structure of the envelopes ejected by the explosions of the classical novae Cyg 1992 and LMC 1991 in the initial expansion period, the constructed models that described best the data, had power-law density profiles with exponents of 15 and 7 , respectively. In our case, a steeper density distribution in the outer parts of the ejected envelope only compounds the situation, because the problem has a constraint on the energy of the outer layers of the envelope. The velocity of the matter at the outer boundary should remain constant, because it determines the temperature at the forward shock.

\footnotetext{
${ }^{1}$ A more accurate (than that in Paper I) calculation of the coefficient in this formula led to a decrease in the energy estimates by a factor of $\sim 2$, i.e., the shock velocity is defined by the formula $D=\frac{2}{3}\left(\frac{E}{A \alpha}\right)^{1 / 3} t^{-1 / 3}$, where $\alpha=2.1$ (Book 1994); the formula for the energy then takes the form $E \sim$ $2.65 \times 10^{42} n_{9} r_{c, 13}^{2} \mathrm{erg}$. Note that this recalculation does not affect the conclusions reached in Paper I in any way.
} 


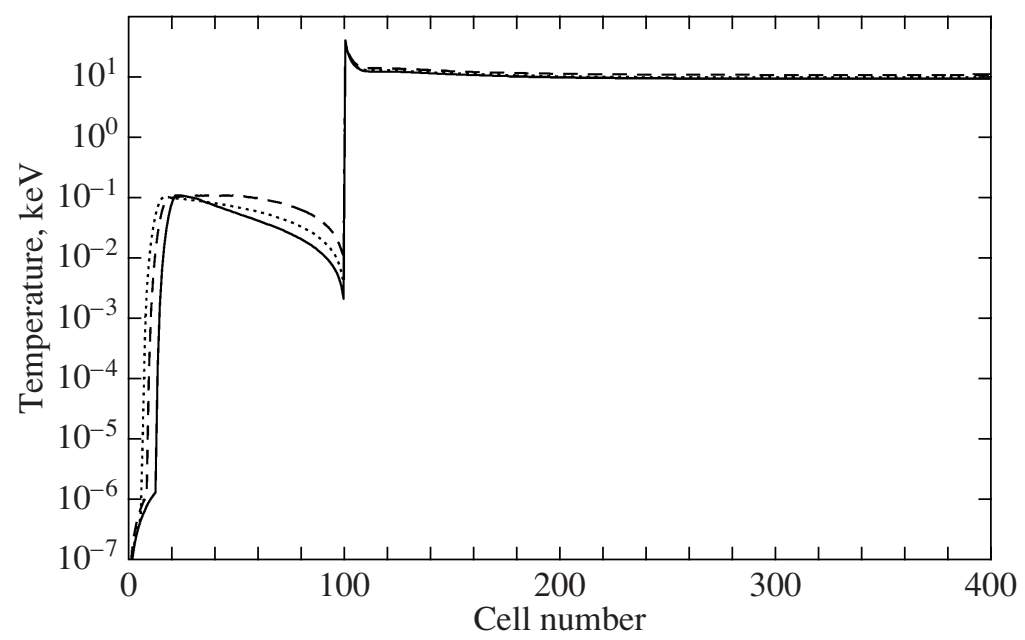

Fig. 5. Temperature profiles behind the forward and reverse shocks on $0.5-0.7$ day after the explosion onset for an envelope with a mass of $\sim 10^{-6} M_{\odot}$ : the solid, dotted, and dashed lines correspond to $\rho=$ const, $\rho \sim r^{-2}$, and $\rho \sim r^{-3}$, respectively. The contact boundary is on cell no. 100 .

Therefore, when the parameter $p$ is varied, the mass of the outer layers with a velocity $>2000 \mathrm{~km} \mathrm{~s}^{-1}$ should be retained. When $p$ increases, this will lead to a decrease in the density at the outer boundary of the ejected matter at the initial time and, hence, the reverse shock will develop more intensively for some time. The dotted line in Fig. 4 indicates the time dependence of the mean temperature for the case where the mass of the matter with a velocity $>2000 \mathrm{~km} \mathrm{~s}^{-1}$ is $2 \times 10^{-5} M_{\odot}$ and $p=15$.

We see from the figure and the above estimates that the case with a homologous expansion of the envelope matter in our one-dimensional model is in conflict with the observational data.

The absence of a homologous expansion phase during the outburst in the system CI Cam can be explained in several ways. The conditions for the generation of a shock wave in the envelope may have not been met during the explosion; therefore, the matter was ejected by thermal pressure without any velocity gradient (Sparks 1969). Or it is possible that the matter ejected as a result of the shock breakout was almost immediately stopped due to the high density of the external medium. The shock produced by it was damped out almost immediately and the observed shock was generated by the subsequent ejection of matter due to the radiation pressure.

\section{ENVELOPE EXPANSION WITH A CONSTANT VELOCITY OF MATTER}

We performed calculations for three density profiles of the matter in the envelope at the initial time: $\rho=$ const, $\rho \sim r^{-2}$, and $\rho \sim r^{-3}$. The envelope mass was taken to be $\sim 10^{-6} M_{\odot}$.
To keep the velocity of the forward shock during the decay of an arbitrary discontinuity the same as that when the piston envelope is pushed, the velocity of the matter in the envelope should be higher than the piston velocity. In these calculations, we set it equal to $3000 \mathrm{~km} \mathrm{~s}^{-1}$.

The derived temperature profiles of the matter behind the forward and reverse shocks are shown in Fig. 5. It follows from this figure that in all cases the temperature behind the reverse shock does not exceed $0.1 \mathrm{keV}$ (recall that radiative cooling in our model switches on at temperatures $>0.1 \mathrm{keV}$ ), i.e., this matter does not contribute to the observed flux in the $3-20 \mathrm{keV}$ energy band and to the temperature averaged over the $\mathrm{X}$-ray flux during approximately the first 0.7 day of expansion.

Investigation of the behavior of the reverse shock at later times is hindered by the absence of reliable theoretical models for the distribution of physical parameters in the expanding matter.

Note that the envelope expanded freely in these calculations. However, as was shown in Paper I, on the first day of expansion, an external force keeping its velocity constant should act on the matter in the envelope. It is quite possible that the reverse shock will be suppressed even more in this case.

We see from the figure that the radiative cooling of the matter behind the reverse shock is important only for the model with a density profile $\rho \sim r^{-3}$ (dashed line) - the temperature profile exhibits a "shelf" downstream of the reverse shock. Let us show that the radiative cooling in this case also takes place in an optically thin regime. 


\section{Radiative Cooling of the Matter Behind the Reverse Shock}

To establish the regime of radiation of the matter downstream of the reverse shock, we calculated its optical depth. The optical depth for Thomson scattering up to the region downstream of the reverse shock in which the radiative cooling becomes important is less than unity. A significant contribution to the absorption of radiation in the envelope matter can be made by absorption in lines. However, using the opacity tables calculated with the OPAL code, we found that for a density of the order of $3 \times$ $10^{12} \mathrm{~cm}^{-3}$ and a temperature of $5 \times 10^{3}-10^{5} \mathrm{~K}$, the absorption cross section in the matter does not exceed $\sim 10^{-24} \mathrm{~cm}^{2}$. The optical depth corresponding to this cross section is also less than unity. Thus, the matter downstream of the reverse shock, just as downstream of the forward shock, radiates in an optically thin regime.

As we have already said above, the heavy-element abundance in the envelope matter ejected by the explosion of a classical nova can be higher than the solar one, which, in turn, can lead to an increase in the cooling rate of the matter. In our calculations, we retained the solar abundance, because the characteristic cooling time for the density obtained in the calculations $\left(3 \times 10^{12} \mathrm{~cm}^{-3}\right)$ is $\tau_{\text {rad }} \sim 4 \mathrm{~s}$ even for the solar abundance. If it is lower by a factor of several, then this will not affect the calculations. It follows from the formulas in Paper I that for the matter downstream of the reverse shock, the characteristic time it takes for a Maxwellian velocity distribution of ions at $T_{i}=0.1 \mathrm{keV}$ to be established is $\tau_{i i} \sim 6 \times 10^{-4} \mathrm{~s}$ (in these estimates, we took the parameters $A_{i}=1, A_{e}=1 / 1836, \ln \Lambda=15, Z_{i}=1$, and $n_{i}=n_{e}$, as in Paper I). Formally, for the ion and electron temperatures $T_{i}=0.1 \mathrm{keV}$ and $T_{e}=1 \mathrm{eV}$, the ion Maxwellization time is longer than the time of temperature equalization between the ions and electrons by a factor of $\tau_{i i} / \tau_{i e}=4.5 \times 10^{-2}\left(T_{e} / T_{i}+\right.$ $\left.5 \times 10^{-4}\right)^{-3 / 2} \sim 42$. This means that the ions initially transfer their energy to the electrons without still having a Maxwellian velocity distribution, while Spitzer's formula for $\tau_{i e}$ begins to work only when $T_{e}>0.13 T_{i} \sim 0.01 \mathrm{keV}$. For our estimations, we take $T_{e}=0.05 \mathrm{keV}$ (in this case, $\tau_{e e} \sim 5 \times 10^{-6} \mathrm{~s}$ ), then $\tau_{i e} \sim 5 \times 10^{-3}$ s. Clearly, the time of electron heating by ions to $T_{e}=0.05 \mathrm{keV}$ should be of the order of the value of $\tau_{i e}$ obtained. The time it takes for an ionization equilibrium to be established is $\tau_{e q} \simeq 1 \mathrm{~s}$. Thus, it follows from our estimates that the characteristic times it take for an equilibrium to be established downstream of the reverse shock are shorter than the characteristic radiative cooling time, $\sim 4 \mathrm{~s}$, and the applicability conditions for the APEC model
(http://hea-www.harvard.edu/APEC/REF) to calculate the plasma energy loss rate are met. ${ }^{2}$

\section{CONCLUSIONS}

We investigated the effect of the reverse shock on the observed parameters of the $\mathrm{X}$-ray emission during the 1998 outburst of CI Cam using a spherically symmetric model for the interaction of the envelope ejected by the nova explosion with the circumstellar matter. Comparison of our numerical calculations and observations within the framework of this model led us to the following conclusions.

(1) The homologous expansion phase of the matter during the explosion in CI Cam most likely was either absent or short and did not give rise to an observable forward shock in the stellar wind. The velocity profile in the matter ejected by the explosion had no steep gradients.

(2) For a free envelope expansion at a constant velocity and the explosion parameters that we obtained in Paper I, the reverse shock could not heat the matter to temperatures above $\sim 0.1 \mathrm{keV}$ during the first $\sim 0.7$ day of expansion.

(3) During the 1998 outburst of CI Cam, the contribution from the matter heated by the reverse shock to the observed luminosity in the $3-20 \mathrm{keV}$ energy band and to the temperature averaged over the X-ray flux during the first $\sim 0.7$ day of envelope expansion was negligible compared to the contribution from the matter behind the forward shock.

\section{ACKNOWLEDGMENTS}

This work was supported by the Russian Foundation for Basic Research (project no. 07-02-01051), the Program of the Russian President for Support of Scientific Schools (NSh-5579.2008.2), and the "Origin, Structure, and Evolution of Objects of the Universe" Program of the Presidium of the Russian Academy of Sciences. E.V. Filippova is also grateful to the Foundation for Support of Russian Science for support. We wish to thank the referees for their useful and important remarks, which helped to improve significantly the paper, and S.A. Grebenev for a discussion of the results obtained.

\footnotetext{
${ }^{2}$ The same reasoning should also applied to the estimates made in Paper I.
} 


\section{REFERENCES}

1. E. A. Barsukova, N. V. Borisov, A. N. Burenkov, et al., Astron. Rep 50, 664 (2006).

2. D. Book, Shock Waves 4, 1 (1994).

3. R. Chevalier, Astrophys. J. 258, 790 (1982).

4. V. Dwarkadas and R. Chevalier, Astron. Astrophys. 497, 807 (1998).

5. E. Filippova, M. Revnivtsev, and A. Lutovinov, Astron. Lett. 34, 797 (2008).

6. M. Friedjung, AIP Conf. Ser. 266 (2002).

7. P. Hauschildt, S. Starrfield, S. Austin, et al., Astrophys. J. 442, 831 (1994).

8. M. Kato and I. Hachisu, Astrophys. J. 437, 802 (1994).

9. F. Khokhlov, E. Müller, and P. Höflich, Astron. Astrophys. 270, 223 (1993).

10. D. McLaughlin, Publ. Astron. Soc. Pacific 59, 244 (1947).

11. D. McLaughlin, Ann. d'Astrophys. 27, 496 (1964).
12. D. Nadyozhin, Astrophys. Space Sci. 112, 225 (1985).

13. D. Prialnik, Astrophys. J. 310, 222 (1986).

14. E. L. Robinson, I. I. Ivans, and W. F. Welsh, Astrophys. J. 565, 1169 (2002).

15. F. K. Röpke, Astron. 432, 969 (2005).

16. G. Schwarz, S. Shore, S. Starrfield, et al., Mon. Not. R. Astron. Soc. 320, 103 (2001).

17. W. M. Sparks, Astrophys. J. 156, 569 (1969).

18. S. Starrfield, W. M. Sparks, and J. W. Truran, in Proc. of the Symp. on Structure and Evolution of Close Binary Systems 73, Ed. by P. Eggleton et al. (Reidel, Dordrecht, 1976), p. 155.

19. S. Woosley and T. Weaver, Ann. Rev. Astron. Astrophys. 24, 205 (1986).

20. O. Yaron, D. Prialnik, M. M. Shara, and A. Kovetz, Astrophys. J. 623, 398 (2005).

Translated by G. Rudnitskii 